\title{
Perceptual learning and the visual control of braking
}

\author{
BRETT R. FAJEN \\ Rensselaer Polytechnic Institute, Troy, New York
}

\begin{abstract}
Performance on a visually guided action may improve with practice because observers become perceptually attuned to more reliable optical information. Fajen and Devaney (2006) investigated perceptual attunement, using an emergency braking task in which subjects waited until the last possible moment before slamming on the brakes. The subjects in that study learned to use more reliable optical variables with practice, allowing them to perform the task more successfully across changes in the size of the approached object and the speed of approach. In Experiment 1 of the present study, subjects completed blocks of normal, regulated braking before and after practice on emergency braking. Size and speed effects that were present at early stages diminished or were eliminated after practice, suggesting that perceptual attunement resulting from practice on emergency braking transfers to normal, regulated braking. In Experiment 2, practice on regulated braking alone also resulted in perceptual attunement. The findings suggest that braking is not always guided on the basis of an optical invariant and that perceptual attunement plays an important role in learning to perform a visually guided action.
\end{abstract}

Why does performance on a visually guided action, such as steering, braking, or fly ball catching, improve with practice? Of course, there are many ways to account for the positive effects of practice. One factor that may contribute to improvement in performance is perceptual attunement - that is, learning to exploit more reliable sources of information. Perceptual attunement has been investigated in the context of perceptual judgment tasks, such as estimating the relative mass of colliding balls (Jacobs, Runeson, \& Michaels, 2001) and judging the length of an unseen rod by means of dynamic touch (Withagen \& Michaels, 2005), as well as discretely controlled perceptual-motor tasks, such as timing the release of a pendulum so as to hit an approaching ball (Smith, Flach, Dittman, \& Stanard, 2001) and timing the initiation of emergency braking to avoid a collision at the last possible moment (Fajen \& Devaney, 2006). The purpose of this study was to extend the investigation of perceptual attunement to the domain of continuously controlled visually guided actions - that is, tasks in which movements are continuously guided on the basis of visual information.

Until recently, the idea that improvement in performance on such visually guided actions can be traced back to perceptual attunement had not been seriously considered. Despite evidence of attunement in perceptual judgment and discretely controlled perceptual-motor tasks, it has typically been assumed that when actors perform a task such as steering, braking, or running to catch a fly ball, their movements are guided by a single optical invariant, regardless of their level of skill. The tau-dot model of visually guided braking (Bardy \& Warren, 1997; Lee,
1976; Yilmaz \& Warren, 1995) provides a useful example illustrating this approach. According to the tau-dot model, braking is guided by making adjustments around a critical value of the optical variable $\dot{\tau}$ ("tau-dot"). $\dot{\tau}$ is the first derivative of the optical variable $\tau$, which is equal to the ratio of the optical angle $\theta$ to the expansion rate $\dot{\theta}$ of the approached object. When $\dot{\tau}$ is less than -0.5 , it indicates that the current rate of deceleration is insufficient and the actor will collide unless deceleration is increased. When $\dot{\tau}$ is greater than -0.5 , deceleration exceeds the rate necessary to avoid a collision, and the observer will stop short of the intended stopping point unless deceleration is reduced. Thus, $\dot{\tau}$ specifies the sufficiency of the actor's current rate of deceleration; and because it does so even across changes in approach speed and object size, $\dot{\tau}$ is classified as an optical invariant. By regulating deceleration around a critical value of $\dot{\tau}$ equal to -0.5 , the observer can effectively null the error between the current deceleration and the deceleration required to stop at the intended stopping point.

The same logic - that actions are guided by making adjustments around the critical value of a single optical invariant - has been applied to the development of models of other continuously controlled visually guided actions, including steering (Wann \& Land, 2000; Wann \& Swapp, 2000), fly ball catching (Chapman, 1968; McBeath, Shaffer, \& Kaiser, 1995; Oudejans, Michaels, Bakker, \& Davids, 1999), interception by hand (Montagne, Laurent, Durey, \& Bootsma, 1999; Peper, Bootsma, Mestre, \& Bakker, 1994), and interception on foot (Chardenon, Montagne, Buekers, \& Laurent, 2002; Fajen \& Warren, 2004;

B. R. Fajen, fajenb@rpi.edu 
Lenoir, Musch, Janssens, Thiery, \& Uyttenhove, 1999). But despite some seemingly convincing support for these models, the assumptions upon which they rest are questionable. The first problem, which Fajen (2005b) referred to as the problem of action boundaries, is that such models fail to capture actors' sensitivity to the most critical constraint on successful performance - that is, the limitations of their action capabilities. In braking, for example, the deceleration required to stop must never exceed the maximum possible deceleration (Fajen, 2005a). Unfortunately, actors relying on $\dot{\tau}$ to control braking would be completely insensitive to this constraint, because $\dot{\tau}$ provides no information about whether the appropriate brake adjustment necessitates exceeding maximum deceleration. Consider, for example, the situation in which an actor is approaching an object at a constant speed - that is, before braking is initiated. If the object is far enough away or if the actor is moving slowly, the deceleration required to stop will be less than maximum deceleration. However, as the actor approaches the object at a constant speed, the deceleration required to stop increases until it eventually exceeds the maximum deceleration, at which point it is no longer possible to avoid a collision by braking. Because $\dot{\tau}$ is always equal to -1.0 whenever approach speed is constant, it tells the actor only that current deceleration (i.e., zero) is insufficient. $\dot{\tau}$ provides no information about when to initiate braking. Even after braking is initiated, $\dot{\tau}$ tells the actor only about the sufficiency of the current deceleration. If the actor is still braking insufficiently, $\dot{\tau}$ does not tell him or her whether the needed deceleration requires exceeding maximum deceleration. In summary, $\dot{\tau}$ fails to provide the information that actors need to determine when to initiate braking and how to modulate braking throughout the approach in a way that takes the limits of their braking capabilities into account. The fact that actors do behave in ways that take these limits into account (see, e.g., Fajen, $2005 \mathrm{a}, 2005 \mathrm{c}$ ) indicates that models such as $\dot{\tau}$ overlook a critical aspect of behavior.

The second problem with the such models, which provided the rationale for the present study, is that they rest on the single optical invariant assumption - the assumption that actions are guided on the basis of the same optical invariant (e.g., $\dot{\tau}$ ), regardless of the availability of information, the range of conditions, and the skill of the actor. The single optical invariant assumption is difficult to reconcile with findings demonstrating biases in performance that are inconsistent with the use of an optical invariant. In braking, for example, Andersen and colleagues (Andersen, Cisneros, Atchley, \& Saidpour, 1999; Andersen \& Sauer, 2004) have reported speed and size effects inconsistent with the use of $\dot{\tau}$, which is invariant across speed and size. Fajen (2005a) found robust effects of the global optic flow rate from the ground plane, which is independent of $\dot{\tau}$. Similar size and speed effects have also been reported in a variety of other collision control tasks, including catching (van der Kamp, Savelsbergh, \& Smeets, 1997), hitting (Michaels, Zeinstra, \& Oudejans, 2001; Smith et al., 2001), and obstacle avoidance (DeLucia \& Warren, 1994). The latter studies have led many investigators to question or reject the single optical invariant assumption in the context of discretely controlled or rapid visually guided actions. Nonetheless, skepticism about the single optical invariant assumption is much less pervasive in studies of continuously controlled visually guided actions. As was indicated above, most models of steering, braking, fly ball catching, and interception on foot continue to adhere to the assumption that movements are guided on the basis of a single, task-specific optical invariant.

A related concern with the single optical invariant assumption is that it leaves no room for the possibility that improvement in performance reflects a process of attunement. Recall from the opening paragraph that evidence of attunement has already been reported in the context of perceptual judgment (Jacobs et al., 2001; Withagen \& Michaels, 2005) and discretely controlled interceptive actions (Fajen \& Devaney, 2006; Smith et al., 2001). In addition, van Hof, van der Kamp, and Savelsbergh (2004, 2006) found that three- to eight-month-old infants learning to catch transition from relying on optical angle to relying on $\tau$ or binocular variables to time the initiation of catching movements. Jacobs and Michaels (2006) investigated the role of attunement in guiding the hand to the interception point during one-handed catching and found changes (albeit subtle ones) in the optical variables on which actors relied.

To summarize, there is compelling evidence from studies of perceptual judgments and discretely controlled (or rapid) interceptive actions that actors do not always rely on optical invariants and that they can learn to rely on more effective variables with practice. Nonetheless, few studies have investigated the role of attunement in the context of continuously controlled visually guided action, and most existing models of continuously controlled visually guided actions continue to subscribe to the assumption that movements are guided on the basis of a single optical invariant. Perhaps one reason why these issues have not been more thoroughly investigated in the context of continuously controlled visually guided actions is that no one has provided an alternative model that could accommodate such findings. Recently, Fajen (2005a, 2005c) introduced an alternative model of visually guided braking that, unlike the tau-dot model, does not require adherence to the single optical invariant assumption. After introducing this model in the next section, I will show how it allows for the possibility that actors rely on noninvariants, as well as learn to rely on more effective information with practice.

\section{The Affordance-Based Control Model}

According to this model, deceleration is controlled not by making adjustments around the critical value of an optical invariant such as $\dot{\tau}$ but, rather, by making adjustments to keep the deceleration required to stop below the maximum rate of deceleration that the actor can produce (or is willing to tolerate). (In practice, actors are likely to keep the deceleration required to stop well below the maximum rate of deceleration in order to allow for a safety margin and to avoid having to slow down at an uncomfortably high rate of deceleration.) In other words, braking is controlled by adjusting deceleration so that it is always 
possible to stop within the actor's actual (or more likely, tolerable) deceleration limits. Because this control strategy relies on the actor's ability to perceive whether safe stopping is afforded, Fajen (2005a, 2007) referred to it as affordance-based control.

To control deceleration so that it is always possible to stop within their deceleration limits, actors must be able to detect and calibrate information about the amount of deceleration required to stop at the intended location, relative to the maximum tolerable deceleration of the brake (hereafter, maximum deceleration). Although there exists an optical invariant that specifies this property, the previous research on visually guided braking (Fajen \& Devaney, 2006), together with research in other collision control tasks (reviewed above), raises doubts that all actors (especially novices) rely on the optical invariant. The present study was designed to test the hypotheses that novices rely on noninvariants and that actors become perceptually attuned to more reliable optical variables (perhaps even the invariant) with practice.

Consider some of the possible optical variables (invariants and noninvariants) on which actors could rely to control braking. In terms of spatial variables, the constant rate of deceleration that would bring the actor to a stop at the intended location is equal to $v^{2} / 2 z$, where $v$ is speed and $z$ is distance. This will be referred to as ideal deceleration. (Ideal deceleration is "ideal" in the sense that no further adjustments are necessary to stop at the intended location, as long as current deceleration is equal to ideal deceleration.) In terms of optical variables, ideal deceleration is specified by GOFR $\times \dot{\theta} / \theta$. GOFR is the global optic flow rate of the textured ground surface, which is proportional to speed as long as eye height is constant, which it typically is in a braking task. ${ }^{1}$ The ratio $\dot{\theta} / \theta$, where $\theta$ and $\dot{\theta}$ are the optical angle and rate of expansion of the approached object, respectively, is approximately equal to $v / z$. Thus, ideal deceleration $\left(v^{2} / 2 z\right)$ can be expressed in terms of optical variables by substituting GOFR for $v$ and $\dot{\theta} / \theta$ for $v / z$ to arrive at GOFR $\times \dot{\theta} / \theta$.

When GOFR $\times \dot{\theta} / \theta$ is properly calibrated to the strength of the brake, it specifies the percentage of maximum deceleration that must be applied to avoid a collision. Furthermore, because it does so across changes in the size of the approached object and the speed of approach, GOFR $\times \dot{\theta} / \theta$ is an optical invariant. This is illustrated in Figures $1 \mathrm{~A}-1 \mathrm{~B}$. The solid line in both figures indicates the value of ideal deceleration at the moment that GOFR $\times \dot{\theta} / \theta$ reaches some arbitrary critical value. Note that ideal deceleration at this moment is the same across variations in both the size of the approached object (Figure 1A) and the speed of approach (Figure 1B). If braking is controlled by making adjustments to keep the ideal deceleration (based on GOFR $\times \dot{\theta} / \theta$ ) below maximum deceleration, performance should be unaffected by changes in size or approach speed.

In addition to GOFR $\times \dot{\theta} / \theta$, there is an infinite number of other optical variables that do not specify ideal deceleration but may still be used to guide braking. Although relying on a noninvariant would lead to systematic biases in performance, it is often possible to correct for errors

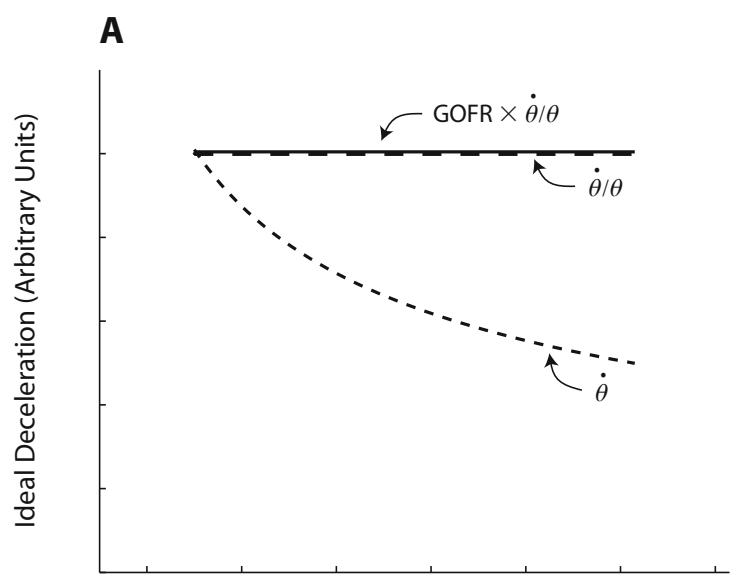

Radius (Arbitrary Units)

\section{B}

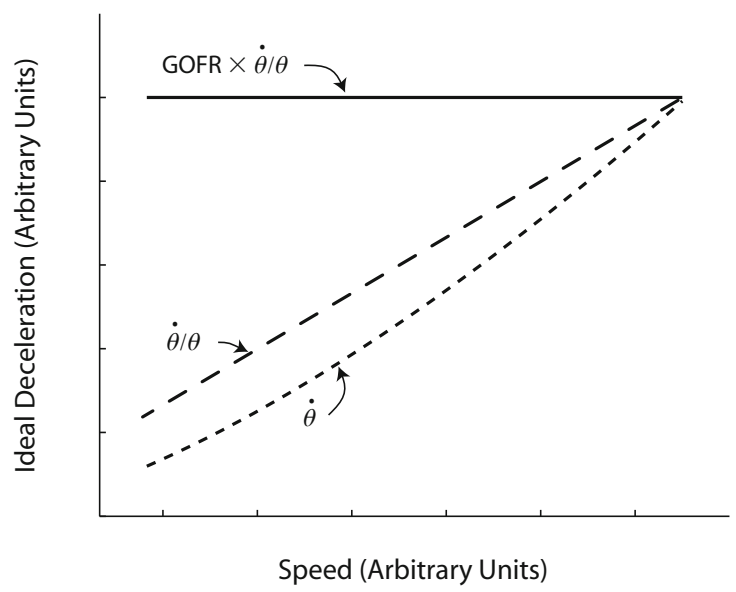

Figure 1. Ideal deceleration at the moment that GOFR $\times \dot{\theta} / \theta$ (solid line), $\dot{\theta} / \theta$ (dashed line), and $\dot{\theta}$ (dotted line) reached an arbitrary critical value, as a function of the radius of the approach object (A) and the speed of approach (B).

that arise from such biases when actions are continuously regulated, as braking usually is. Furthermore, the reliability of noninvariants depends on the range of sizes and approach speeds, and some noninvariants are more reliable than others. So, depending on the range of conditions and the tolerance for error, performance based on a noninvariant may be acceptable.

A simple example of a noninvariant for braking is $\dot{\theta} . \dot{\theta}$ correlates with, but does not specify, the percentage of maximum deceleration that must be applied to avoid a collision. The dotted line in Figure 1A shows the value of ideal deceleration at the moment that $\dot{\theta}$ reaches an arbitrary critical value as a function of radius. The negative slope indicates that actors relying on $\dot{\theta}$ would brake earlier and/or harder than necessary when the size of the approached object is large. Likewise, the positive slope 
in Figure 1B indicates that actors relying on $\dot{\theta}$ would also be more likely to brake earlier and/or harder than necessary when approach speed is slow. Another example of an optical variable is $\dot{\theta} / \theta$, which is invariant over changes in size, but not in speed (see the dashed lines in Figures $1 \mathrm{~A}$ and $1 \mathrm{~B}$ ). Actors relying on $\dot{\theta} / \theta$ would be unaffected by manipulations of the size of the approached object but would brake earlier and/or harder than necessary when approach speed is slow.

Figure 1 illustrates that the patterns of size and speed biases that subjects exhibit can be used to infer the optical variables on which they rely. However, it is important to keep in mind that $\dot{\theta}, \dot{\theta} / \theta$, and GOFR $\times \dot{\theta} / \theta$ are simply three out of an infinite number of optical variables and that actors may also rely on variables that cannot be expressed in terms of simple combinations of $\theta, \dot{\theta}$, and GOFR. Thus, actual data may not necessarily conform to the predictions of one of these three variables.

Fajen and Devaney (2006) recently demonstrated that people come to exploit more reliable optical variables with practice on a modified braking task, which will be referred to as the emergency braking task. Subjects watching computer simulations of linear self-motion toward a stop sign were instructed to wait until the last possible moment before slamming on the brakes. Braking was initiated by pulling back on a joystick, at which point a constant rate of deceleration of $10 \mathrm{~m} / \mathrm{sec}^{2}$ was applied until the actor's simulated speed reached zero. Because deceleration could not be adjusted after braking had been initiated, the emergency braking task required one to learn when to initiate deceleration. Sign radius and initial speed were manipulated to determine the optical variables upon which the observers relied at various stages of practice. Trials were presented in blocks of 30, and separate analyses were performed on the data from each block. Early in practice, effects of sign radius and initial speed consistent with the use of a noninvariant were found. Novices tended to initiate emergency braking earlier than necessary when initial speed was slow and, to a lesser extent, when the sign radius was large. With additional practice, the weak size effect was quickly eliminated, and the speed effect diminished but was not eliminated. The absence of a size effect in practiced subjects rules out the possibility that the subjects relied on $\dot{\theta}$ but does not, by itself, allow us to determine which of the size-invariant optical variables (e.g., $\dot{\theta} / \theta$, GOFR $\times \dot{\theta} / \theta$, or some other size-invariant variable) the subjects actually used. However, the speed effect was weaker than that predicted by $\dot{\theta} / \theta$, and as it diminished with practice, the data converged toward the predictions of GOFR $\times \dot{\theta} / \theta$ (see Figure 1B). Taken together, the results suggest that the subjects learned to use GOFR together with $\theta$ and $\dot{\theta}$ in a way that resembled the optical invariant.

The starting point for the present study was to determine whether the findings of Fajen and Devaney (2006) would generalize to normal, regulated braking. Although the emergency braking task is unlike normal, real-world braking, in which ongoing adjustments are possible, there are important similarities between these two tasks. To perform the emergency braking task, subjects must initiate braking at the exact moment that ideal deceleration reaches the fixed constant rate of deceleration of the brake. Thus, performance on the emergency braking task provides an indication of the reliability with which actors detect and calibrate information about ideal deceleration across changes in size and speed. If normal, regulated braking is controlled by keeping the ideal deceleration within the limits of the brake, as is suggested by the affordance-based control model, and if observers rely on noninvariants, as was suggested by Fajen and Devaney, subjects should exhibit biases in normal, regulated braking that are consistent with the biases exhibited in emergency braking. That is, novices should tend to brake earlier and/or harder when initial speed is slow and, to a lesser extent, when the sign radius is large. Furthermore, practice on emergency braking that results in perceptual attunement to more reliable optical variables should result in less biased performance on normal regulated braking. Such findings would have interesting theoretical implications, insofar as they would contradict the single optical invariant assumption (on which the tau-dot model is based) and would imply that models of visually guided braking must include a role for perceptual attunement.

In Experiment 1, blocks of emergency braking were interleaved with blocks of normal, regulated braking. If the perceptual attunement that occurs during emergency braking transfers to normal, regulated braking, novices should exhibit biases consistent with those reported by Fajen and Devaney (2006), and these biases should diminish following practice on emergency braking. The purpose of Experiment 2 was to determine whether perceptual attunement also occurs during normal, regulated braking. Thus, subjects performed blocks of regulated braking only, and performance was compared across blocks.

\section{EXPERIMENT 1}

Subjects completed 11 blocks of trials, and each block consisted of 25 trials. Three blocks of regulated braking $(1,6$, and 11$)$ were interleaved with 8 blocks of emergency braking (2-5 and 7-10). Within each block, there were five sign radii and five initial speeds. The dependent variables were ideal deceleration at the onset of braking, initial brake adjustment magnitude, and final stopping distance. The influence of available information on perceptual attunement was also tested by manipulating the presence of the textured ground plane as a between-subjects factor. The subjects in the ground condition performed the task with the textured ground plane present, and those in the air condition performed the task with the ground plane absent.

\section{Method}

Participants. Twenty-four students (21 men, 3 women) participated in Experiment 1. Half were assigned to the ground condition, and the other half to the air condition. All had normal or correctedto-normal vision and at least 1 year of driving experience.

Displays and Apparatus. Displays were generated using OpenGL running on a Dell Precision 530 Workstation and were rear-projected by a Barco Cine 8 CRT projector onto a large $(1.8 \times 1.2 \mathrm{~m})$ screen at a frame rate of $60 \mathrm{~Hz}$. The displays simulated observer movement along a linear path toward three red-and-white octagonal stop signs (see Figure 2A). The sky was light blue, and a gray cement-textured ground surface $1.1 \mathrm{~m}$ below the observer's viewpoint was present in 
A

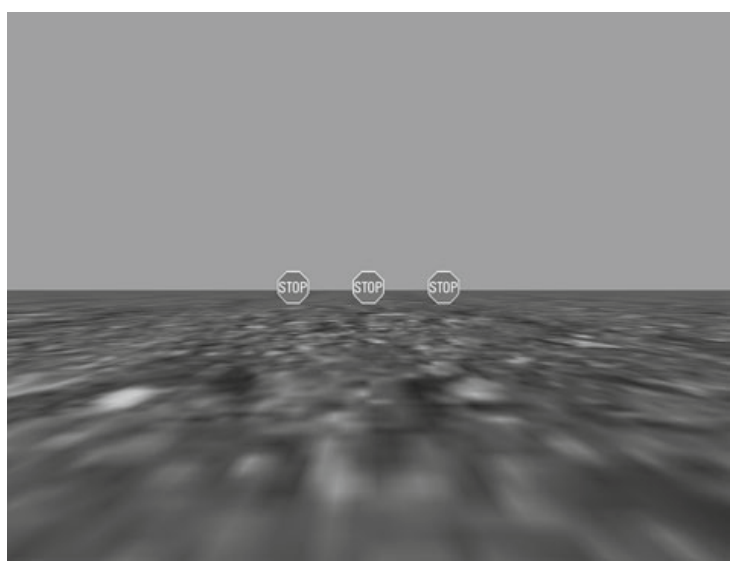

B

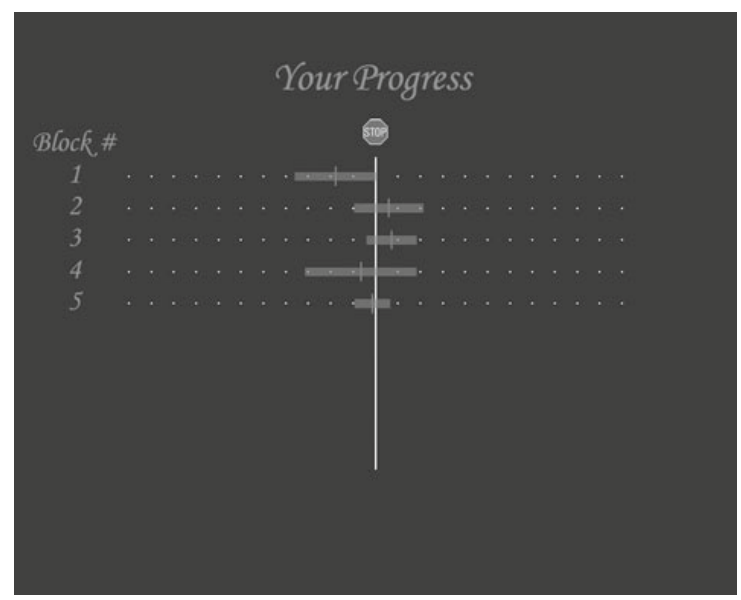

Figure 2. (A) Sample screen shot of displays used in the ground condition in both experiments. (B) Performance summary screen shown at end of each block.

the ground condition, but not in the air condition. One stop sign was positioned on the observer's simulated path of motion, and the other two were positioned on the right and left. The distance between the stop signs was always four times the radius of the signs. The center of each sign was at the same height as the simulated viewpoint, and there were no posts anchoring the bottom of the signs to the ground surface. Posts were left out of the simulated scene to provide a stronger test of the size manipulation. If posts were present, the distance from the center of the stop sign to the ground would be constant, even across changes in stop sign radius.

Braking was controlled using a Microsoft Sidewinder Force Feedback 2 joystick. The subjects increased deceleration by pulling the joystick from the neutral center position. In regulated braking blocks, the brake was programmed so that deceleration was proportional to joystick position. Deceleration ranged from $0 \mathrm{~m} / \mathrm{sec}^{2}$ in the neutral center position to $10 \mathrm{~m} / \mathrm{sec}^{2}$ in the downmost position. In the emergency braking blocks, a fixed rate of deceleration of $10 \mathrm{~m} / \mathrm{sec}^{2}$ was triggered at the moment that the joystick was displaced from the center position.

At the end of each block, a screen summarizing the subject's performance on each completed block was presented (see Figure 2B).
The white vertical line under the stop sign corresponded to the location of the stop sign. Mean stopping location for each block was indicated by the short gray line (red in the actual display), and the standard deviation of stopping distance was indicated by the gray bar (blue in the actual display).

Design. There were five values for the sign radius $(0.165,0.195$, $0.255,0.375$, and $0.615 \mathrm{~m})$ and five values of initial speed $(8,10$, 12,14 , and $16 \mathrm{~m} / \mathrm{sec}$ ). Initial distance varied randomly between 50 and $60 \mathrm{~m}$ during regulated braking and between 40 and $50 \mathrm{~m}$ during emergency braking. Within each block, there was one repetition per condition, for a total of 25 trials per block. Each subject completed 11 blocks. Blocks 1, 6, and 11 were regulated braking blocks, and Blocks $2-5$ and $7-10$ were emergency braking blocks. Prior to the experiment, the subjects performed 2 practice trials of regulated braking and 2 practice trials of emergency braking, with the experimenter present, in order to familiarize themselves with the task.

Procedure. Trials were initiated by moving the joystick to the neutral, zero-deceleration position and pressing the trigger button on the joystick. The scene appeared, and simulated motion toward the stop signs began immediately. In the regulated braking blocks, the subjects were given specific instructions to encourage smooth braking. They were told to imagine that they were driving an actual automobile, where hard or jerky brake adjustments would be uncomfortable and unsafe. To discourage the subjects from waiting until the last possible moment before initiating full brake pressure, the simulation played a skidding sound whenever brake pressure exceeded $9.5 \mathrm{~m} / \mathrm{sec}^{2}$. In the emergency braking blocks, the subjects were told that the brake was not like a normal brake, in that deceleration could not be adjusted once braking was initiated. Hence, their task was to figure out when to start braking so that they would stop as closely as possible to the stop signs. There was no skid warning during emergency braking blocks.

Displays ended when the subjects came to a stop. Even if they collided with the stop sign, the trial continued until speed was equal to zero. To indicate that a collision had occurred, an audio signal (a beep) lasting $1 \mathrm{sec}$ was played upon impact with the stop sign. The final frame was displayed for $1 \mathrm{sec}$ before the intertrial screen appeared. The subjects were encouraged to monitor their performance at the end of each block and to keep trying to improve performance throughout the entire experiment. The entire experiment lasted less than $1 \mathrm{~h}$.

Data analyses. In emergency braking blocks, the onset of braking was determined simply by identifying the frame at which deceleration was initiated. In regulated braking blocks, the onset of braking was determined by looking for the first window lasting $200 \mathrm{msec}(12$ frames) over which deceleration changed by at least $0.5 \mathrm{~m} / \mathrm{sec}^{2}(5 \%$ of maximum deceleration). Ideal deceleration at onset was calculated using the following equation: $\left(v_{\text {onset }}\right)^{2} /\left(2 \times z_{\text {onset }}\right)$, where $v_{\text {onset }}$ and $z_{\text {onset }}$ were the speed and distance at the onset of braking. The end of the initial brake adjustment was found by searching for the first $200 \mathrm{msec}$ window after brake onset during which deceleration was either held constant ( $\pm 1 \%$ of maximum deceleration) or decreased. The magnitude of each initial brake adjustment was calculated by first taking the change in deceleration from the beginning to the end of the adjustment. The change in deceleration was then scaled to the change in deceleration that was required to null the difference between current and ideal deceleration. Thus, when the initial brake adjustment resulted in an overshoot (or undershoot), the scaled magnitude of adjustment was greater (or less) than 1.0. ${ }^{2}$ For example, if the observer increased deceleration from 0 to $3 \mathrm{~m} / \mathrm{sec}^{2}$ and the ideal deceleration at the end of the initial adjustment was $4 \mathrm{~m} / \mathrm{sec}^{2}$, the scaled magnitude of adjustment would be 0.75 . This indicates that the participant undershot by $25 \%$.

\section{Results and Discussion}

The effects of size and speed were analyzed separately because there was only one data point per block for each combination of size and speed. Statistical analyses were performed using the first and last blocks of regulated 
braking (Blocks 1 and 11) and the first and last blocks of emergency braking (Blocks 2 and 10).

Size effect. Figures $3 \mathrm{~A}-3 \mathrm{~B}$ show the mean ideal deceleration at the onset of braking in Blocks 2 and 10 - the first and last emergency braking blocks. In the ground condition (Figure 3A), neither the main effect of sign radius $[F(4,44)=1.50, p=.24]$ nor the sign radius $\times$ block interaction $[F(4,44)=1.49, p=.24]$ was significant, suggesting that the subjects were attuned to a size-invariant optical variable at the beginning and end of the experiment. In the air condition (Figure $3 \mathrm{~B}$ ), the sign radius $\times$ block interaction was significant $[F(4,40)=4.12, p<$ $.05] .^{3}$ The simple main effect of sign radius was weak but significant in Block 2, but nonsignificant in Block 10 . This pattern of results suggests that the subjects in the air condition were not initially attuned to a size-invariant optical variable but learned to use one with practice.

The main prediction in this experiment was that performance in the first and last regulated braking blocks would be consistent with performance in the first and last emergency braking blocks. Figures $3 \mathrm{C}-3 \mathrm{D}$ show the mean ideal deceleration at the onset of braking in the first and last regulated braking blocks (Blocks 1 and 11). Analyses revealed a significant effect of sign radius in both the ground condition $[F(4,44)=9.27, p<.01$; see Figure $3 \mathrm{C}]$ and the air condition $[F(4,40)=4.17, p<.05$; see Figure $3 \mathrm{D}]$ but no sign radius $\times$ block interaction. These results seem to suggest that the subjects in both conditions were attuned to the same noninvariant at the beginning and end of the experiment. This is inconsistent with the results from the first and last emergency braking blocks and would seem to undermine the main hypothesis of the experiment. However, there is an alternative interpretation of the size effect on ideal deceleration at onset. In regulated braking blocks, deceleration was initiated much earlier than it was in emergency braking blocks because the subjects were told to make smooth, moderate brake adjustments and to avoid having to slam on the brakes. Thus, the distance to the stop signs at the moment that braking was initiated was longer for regulated braking than for emergency braking. At such distances, small stop signs expand at very slow rates. So another possible interpretation of the size effect on ideal deceleration at onset is that the subjects waited longer when the sign radius was small, because optical expansion rate was less salient.

To further explore the size effect, an analysis of the magnitude of the initial brake adjustment was conducted (Figures 3E-3F). If, in fact, the size effect described above was due to the use of a noninvariant, there should also be a tendency to brake harder when the sign radius is large. (Recall that the magnitude of adjustment was calculated so that the change in deceleration during each adjustment was scaled to the required change in deceleration. Thus, a magnitude of adjustment greater than 1.0 indicates an overshoot and less than 1.0 indicates an undershoot. See the Method section for additional details.) In the ground condition (Figure 3E), neither the sign radius effect $(F<1)$ nor the sign radius $\times$ block interaction $(F<1)$ was significant. In the air condition (Figure $3 F$ ), there was a weak trend in Block 1 that disappeared in Block 11, but neither the sign radius effect $(F<1)$ nor the sign radius $\times$ block interaction $(F<1)$ was significant. These results are more consistent with those of the first and last emergency braking blocks: The subjects in the ground condition appear to have been using a size-invariant optical variable; those in the air condition learned to use a size-invariant variable with practice.

The analyses described above were concerned with the initial brake adjustment. To determine whether these effects persisted throughout the entire approach, an analysis of final stopping distance was performed. In the ground condition (Figure $3 \mathrm{G}$ ), neither the main effect of sign radius $[F(4,44)=1.30, p=.29]$ nor the sign radius $\times$ block interaction $[F(4,44)=2.06, p=.15]$ was significant. In the air condition, the interaction was significant $[F(4,40)=3.50, p<.05]$, revealing a weak size effect that disappeared with practice (Figure $3 \mathrm{H}$ ).

Speed effect. The mean ideal deceleration at the onset of braking in the first and last emergency braking blocks is shown in Figures 4A-4B. Statistical analyses revealed a significant speed $\times$ block interaction in the ground condition $[F(4,44)=4.01, p<.05]$, but not in the air condition $[F(4,40)=2.95, p=.06]$. In the ground condition (Figure $4 \mathrm{~A})$, the pattern of results reveals an effect of speed that diminished but was not eliminated with practice. The effect of speed was initially stronger in the air condition (Figure 4B) than in the ground condition and did not significantly change with practice.

The pattern of results in the first and last regulated braking blocks was nearly identical. For the analysis of ideal deceleration at onset (Figures 4C-4D), there was a significant effect of initial speed that changed with practice in the ground condition $[F(4,44)=6.68, p<.01$, for the speed $\times$ block interaction; see Figure $4 \mathrm{C}]$ and a stronger effect that did not change in the air condition $(F<1$; see Figure 4D). The analysis of brake adjustment magnitude was also consistent. The speed $\times$ block interaction was significant in the ground condition $[F(4,44)=5.30, p<$ .01 ; see Figure 4E], but not in the air condition $(F<1$; see Figure 4F). Finally, the analysis of final stopping distance revealed a similar pattern of results $[F(4,44)=4.91, p<$ .01 , for the speed $\times$ block interaction in the ground condition; $F<1$ in the air condition; see Figures $4 \mathrm{G}-4 \mathrm{H}$ ], confirming that the effect of speed continued to influence braking after the initial adjustment. Taken together, analyses of the speed effect suggest that the subjects learned to use GOFR when it was available to perform the task more successfully across changes in speed. However, because the speed effect persisted through Block 11, it appears that GOFR was not used in a manner that was perfectly consistent with the optical invariant.

To summarize, the results indicate that performance on a visually guided braking task is biased by manipulations of speed and (to a lesser extent) size. Such biases are inconsistent with the tau-dot model of braking and, more generally, with the single optical invariant assumption but are consistent with the effects reported by Fajen and Devaney (2006) and replicated here using an emergency braking task. Thus, the present study shows that the biases observed in emergency braking generalize to 

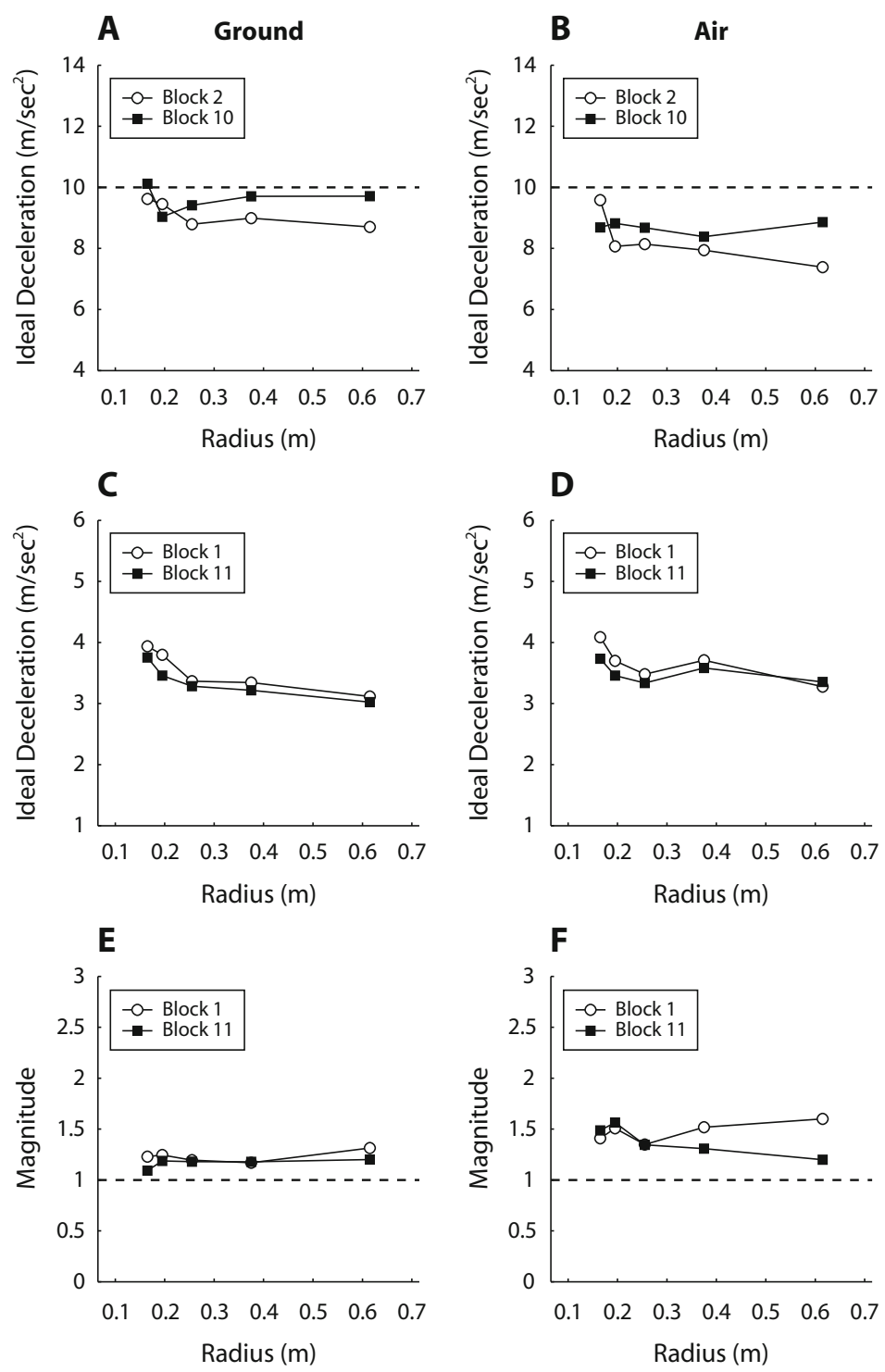

\section{G}
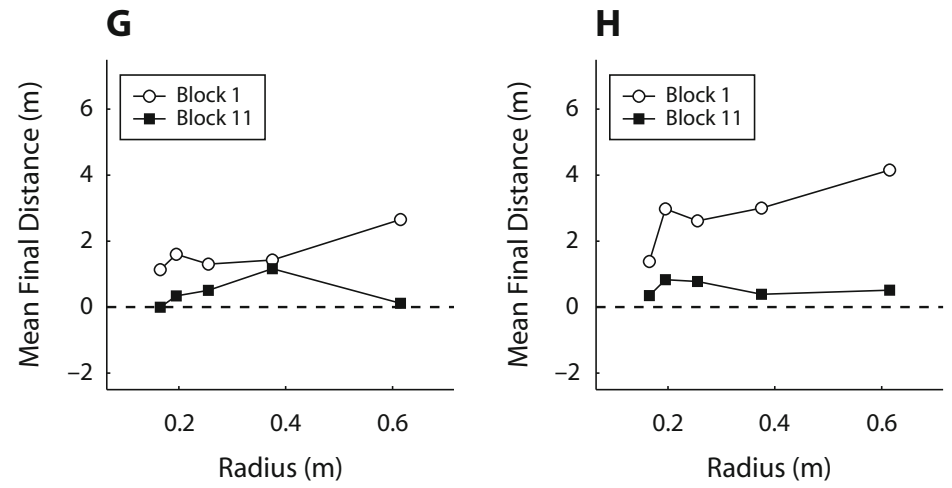

Figure 3. Data from Experiment 1 in the ground (left column) and air (right column) conditions as a function of size. (A-B) Ideal deceleration at the onset of emergency braking. (C-D) Ideal deceleration at the onset of regulated braking. (E-F) Magnitude of initial adjustment during regulated braking. (G-H) Final stopping distance during regulated braking. 

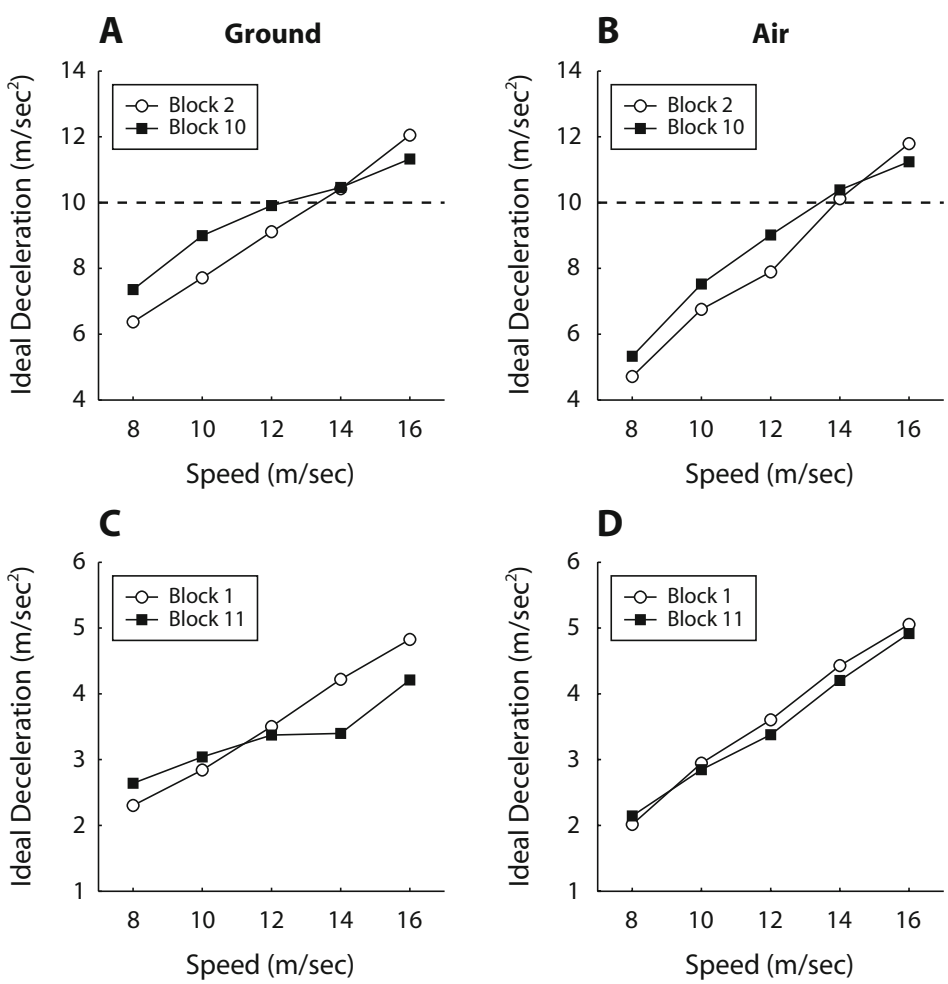

D
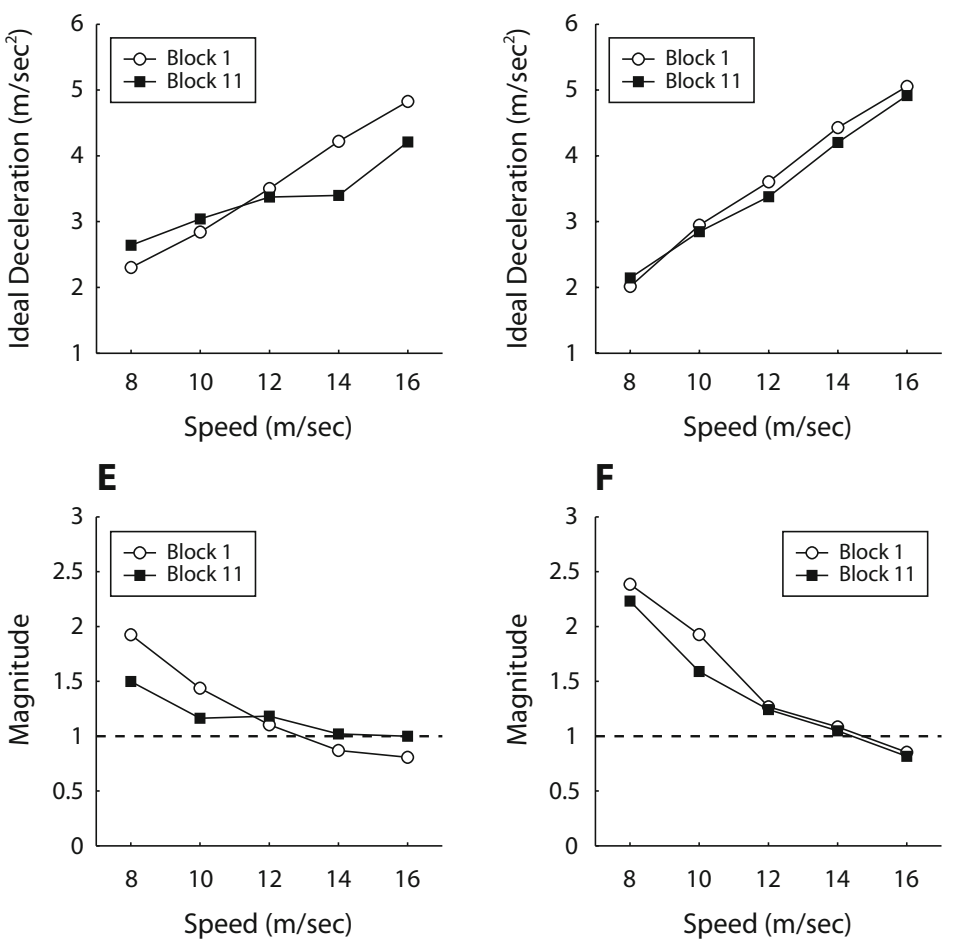

G
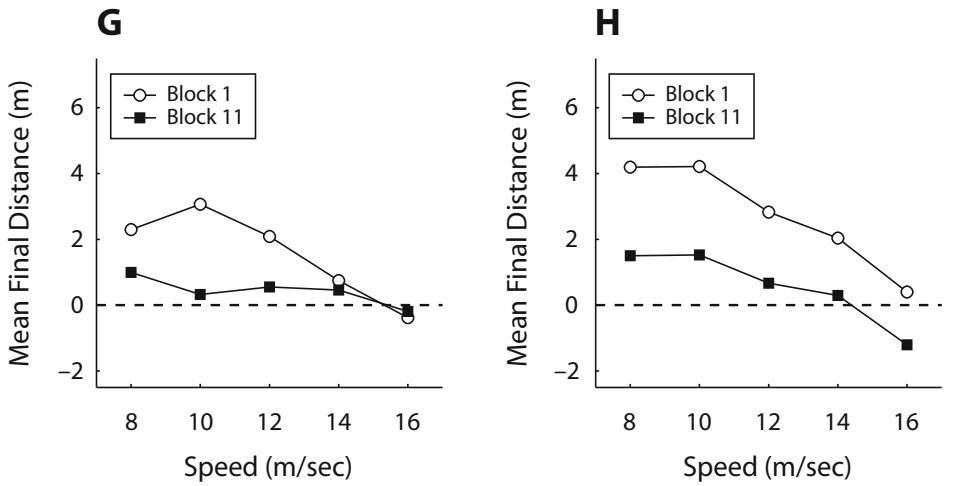

Figure 4. Data from Experiment 1 in the ground (left column) and air (right column) conditions as a function of speed. (A-B) Ideal deceleration at the onset of emergency braking. (C-D) Ideal deceleration at the onset of regulated braking. (E-F) Magnitude of initial adjustment during regulated braking. (G-H) Final stopping distance during regulated braking. 
normal, regulated braking. The second major finding was that perceptual attunement resulting from practice on the emergency braking task transferred to normal, regulated braking. In the air condition, the weak bias to brake earlier and harder for larger stop signs was eliminated with practice on emergency braking, suggesting that the subjects learned to rely on a size-invariant optical variable with practice. The bias to brake earlier (i.e., at lower values of ideal deceleration) and harder when initial speed is slow was weaker after practice with emergency braking, but only in the ground condition, suggesting that the subjects learned to use GOFR (together with $\theta$ and $\dot{\theta}$ ) when it was available to perform the task more reliably across changes in speed. The pattern of results was not restricted to measures based on the initial brake adjustment but applied also to final stopping distance, suggesting that the effects of size and speed continued to influence braking in a consistent manner throughout the entire approach.

Comparing the pattern of size and speed effects with the predictions in Figure 1 allows us to draw some conclusions about the specific optical variables on which actors relied at different stages of practice. For inexperienced subjects, the size effect was initially weak or absent, and the speed effect was strong in the ground condition and even stronger in the air condition. Such effects are most consistent with the use of $\dot{\theta} / \theta$ by inexperienced subjects. (Inexperienced subjects in the ground condition may have been weakly attuned to GOFR as well, accounting for the small difference in the magnitude of the speed effect between the ground and air conditions.) The fact that the speed effect changed with practice, but only for the subjects in the ground condition, indicates that the subjects converged from $\dot{\theta} / \theta$ toward the optical invariant $(\mathrm{GOFR} \times \dot{\theta} / \theta)$ with experience.

\section{EXPERIMENT 2}

In Experiment 1, the subjects learned to use more reliable optical variables by practicing on the emergency braking task. In the real world, people do not normally brake by waiting until the last possible moment before slamming on the brakes. The purpose of Experiment 2 was to determine whether perceptual attunement occurs also during normal, regulated braking. The subjects completed 11 blocks of 25 trials under normal, regulated braking conditions. As in Experiment 1, there were five sign radii and five initial speeds, and the presence of the textured ground plane was manipulated as a between-subjects factor.

\section{Method}

Participants. Twenty different students ( 10 men, 10 women) participated in Experiment 2. Ten students were randomly assigned to the ground condition, and the other 10 to the air condition. All had normal or corrected-to-normal vision and at least 1 year of driving experience.

Displays, Design, and Procedure. The displays, design, and procedure were similar to those used in Experiment 1, with the exception that all 11 blocks were regulated braking

\section{Results and Discussion}

Size effect. The effects of size closely mirrored those in Experiment 1. In both the ground and the air condi- tions, braking was initiated significantly later when the sign radius was small (see Figures 5A-5B). The block $\times$ sign radius interaction was nonsignificant in the ground condition $(F<1)$ but did reach significance in the air condition $[F(4,32)=3.15, p<.05] .{ }^{4}$ Recall that in Experiment 1 , the size effect was interpreted as being due to slower, less salient optical expansion rates associated with smaller stop signs viewed at large distances. Support for this interpretation was provided by the analysis of brake adjustment magnitude. In Experiment 2, the results were similar. In both the ground (Figure 5C) and the air (Figure 5D) conditions, the effect of sign radius on the magnitude of the initial brake adjustment was not significant [ground, $F(4,32)=2.17, p=.10$; air, $F<1$ ] and did not change with practice [ground, $F(4,32)=1.89, p=.17$; air, $F<1]$.

The design of Experiment 2 allowed for the analysis of subsequent brake adjustments that were executed after the initial adjustment. If, in fact, the subjects were attuned to a size-invariant optical variable, the mean ideal deceleration at which subsequent brake adjustments were initiated should also be unaffected by sign radius. Such an analysis was not possible in Experiment 1, due to the small total number of regulated braking trials (i.e., 75 trials in Experiment 1, as compared with 275 in Experiment 2). More trials are necessary for the analysis of subsequent brake adjustments because subjects sometimes make only one identifiable adjustment per trial. Because the size analyses described above revealed only minor changes with practice, the data from Experiment 2 could be collapsed across blocks to allow for a more reliable analysis of the effects of size on subsequent adjustments.

The analysis of subsequent brake adjustments focused on adjustments that resulted in an increase in deceleration, because adjustments that resulted in a decrease were more difficult to clearly identify from the deceleration time series. Although there was a weak trend in the ground condition, the effect of sign radius on the ideal deceleration at the onset of subsequent adjustments did not reach significance in either condition $[F(4,32)=2.25, p=.13$, in the ground condition; $F(4,32)=1.04, p=.36$, in the air condition]. The absence of a significant size effect on subsequent brake adjustments provides further evidence that the subjects in both conditions were attuned to an optical variable that was invariant (or nearly so) across changes in size. This finding is also inconsistent with models of visually guided braking that are based on perceived distance estimated from familiar size. For example, Andersen et al. (1999; Andersen \& Sauer, 2004) suggested that braking is controlled by comparing the estimated distance to the object with the estimated distance that one would travel if deceleration were held constant. The difference between estimates tells the actor whether to increase or decrease deceleration. They proposed that distance to the object was estimated on the basis of familiar size. However, if perceived distance based on familiar size is used to control braking, one would expect manipulations of size to influence braking. Thus, the absence of size effects in this study is inconsistent with Andersen's constant deceleration model as it was originally proposed. A variation of this model that relies on other dis- 

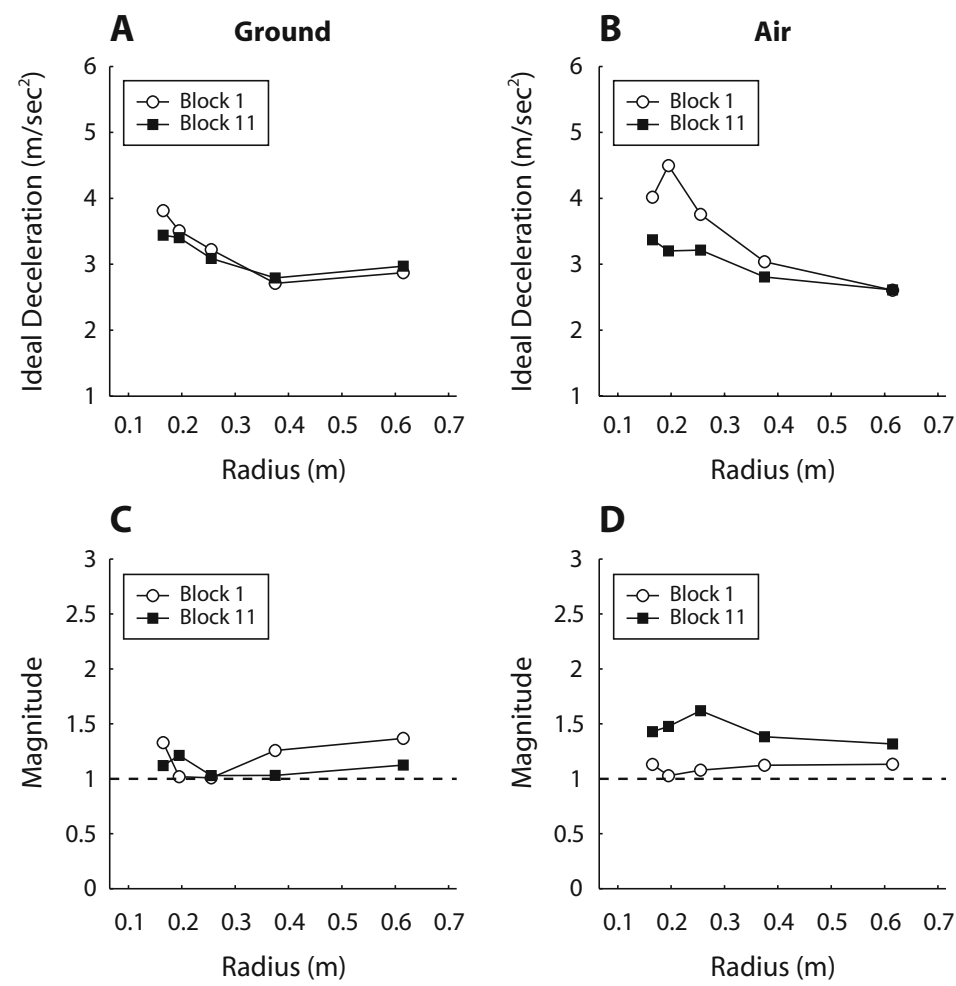

Figure 5. Data from Experiment 2 in the ground (left column) and air (right column) conditions as a function of size. (A-B) Ideal deceleration at the onset of braking. (C-D) Magnitude of initial adjustment.

tance cues could account for the absence of a size effect. However, it is not clear how this model could capture other aspects of these data, such as improvement in performance due to perceptual attunement.

Speed effect. Figures 6A-6B show the mean ideal deceleration at the onset of braking as a function of initial speed for the first and last blocks. As in Experiment 1, there was a robust effect of initial speed in both conditions. This effect changed with practice in the ground condition $[F(4,32)=2.70, p=.06$; see Figure 6A], but not in the air condition $[F(4,32)=1.95, p=.16$; see Figure $6 \mathrm{~B}]$. The analysis of initial brake adjustment magnitude provides further evidence that the subjects in the ground condition learned to use GOFR, since the block $\times$ speed interaction was significant in the ground condition $[F(4,32)=$ $6.85, p<.01$; see Figure $6 \mathrm{C}]$, but not in the air condition $(F<1$; see Figure 6D).

Unfortunately, an analysis of speed effects on subsequent brake adjustments was not possible because, unlike size, speed changes within the trial after braking is initiated. Furthermore, because the speed effect changed with practice, it was not possible to collapse the data across blocks. Thus, the analysis of speed effects is restricted to the initial adjustment.

To summarize, the results of Experiment 2 indicate that practice on regulated braking also led to perceptual attunement and that the degree to which the subjects learned was comparable to the degree to which they learned with practice on emergency braking in Experiment 1. Overall, the pattern of results is consistent with the conclusion from Experiment 1 that inexperienced subjects relied on $\dot{\theta} / \theta$ but converged toward the optical invariant with practice. It is interesting to note that performance was still affected by speed at the end of both experiments. Thus, although practiced subjects were attuned to a size-invariant optical variable and learned to use GOFR when it was available, they did not appear to be relying on the optical invariant. In the present study, the subjects practiced for less than $1 \mathrm{~h}$. It is not known whether additional practice would result in weaker performance biases. Nor is it known whether subjects can learn to rely on the optical invariant, or what kinds of practice conditions facilitate perceptual attunement. Further research on perceptual attunement in the context of visually guided action is needed to answer these questions.

\section{GENERAL DISCUSSION}

The goal of this study was to investigate the role of perceptual attunement in visually guided action. Two significant findings were reported that bear upon this issue. First, braking behavior was biased by approach speed and, to a lesser extent, sign radius. In both experiments, the subjects initiated braking earlier (i.e., at lower values of ideal 

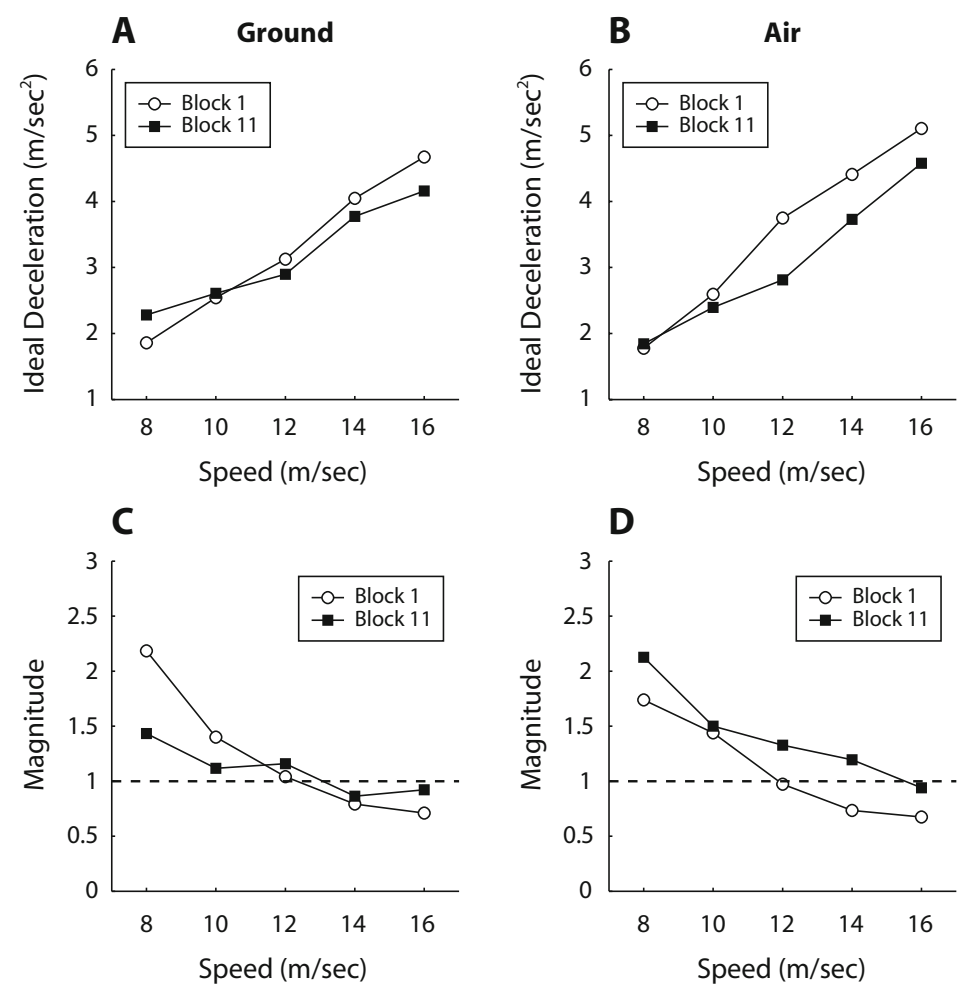

Figure 6. Data from Experiment 2 in the ground (left column) and air (right column) conditions as a function of speed. (A-B) Ideal deceleration at the onset of braking. (C-D) Magnitude of initial adjustment.

deceleration) when the approach speed was slow and, under some conditions, when the sign radius was large. These effects continued to influence braking behavior throughout the entire approach, resulting in biases in final stopping position. Second, these effects diminished with practice under some conditions. In Experiment 1, the subjects performed a regulated braking task before and after practice on an emergency braking task that had previously been shown to result in perceptual attunement (Fajen \& Devaney, 2006). Size and speed effects that were present before practice were weaker or were eliminated after practice on the emergency braking task, suggesting that the perceptual attunement that occurs during emergency braking transfers to normal, regulated braking. In Experiment 2, it was shown that practice on regulated braking also resulted in perceptual attunement.

The pattern of results suggests that the changes in perceptual attunement that were observed in this study can be roughly characterized in terms of a convergence from $\dot{\theta} / \theta$ by inexperienced subjects toward GOFR $\times \dot{\theta} / \theta$ by experienced subjects. This means that for novice subjects, variations in GOFR produced very little change in perceived ideal deceleration. The deceleration needed to stop at a target $20 \mathrm{~m}$ away when moving at a speed of $10 \mathrm{~m} / \mathrm{sec}$ would be incorrectly perceived as the same as the deceleration needed to stop at a target $10 \mathrm{~m}$ away when moving at a speed of $5 \mathrm{~m} / \mathrm{sec}$. (In fact, ideal deceleration is greater in the first situation by a factor of two.) The subjects in the present study learned to use GOFR when it was available. But even after practice, changes in GOFR resulted in less than proportional changes in perceived ideal deceleration. In the example above, a practiced subject would perceive that ideal deceleration is greater in the first case, but by a factor of less than two.

These findings are incompatible with the single optical invariant assumption - a core assumption upon which models of visually guided action, such as the tau-dot model, are based. Because optical invariants are, by their very nature, invariant across changes in conditions, the single optical invariant assumption predicts that performance should not be biased by the manipulation of sign radius and approach speed. Moreover, if each kind of task (e.g., braking, steering) is always controlled on the basis of one and only one optical invariant, both novices and experts should rely on the same optical variables. Neither prediction was supported by the data.

The affordance-based model of braking that was presented in the introduction provides a plausible alternative to the tau-dot model within which the present findings can be more easily understood. The affordance-based model asserts that braking is controlled, not by nulling the error between the current and the ideal deceleration, but by keeping ideal deceleration within the safe region between zero and maximum deceleration - that is, by braking so 
that it is always possible to stop within the actual or tolerable deceleration limits. This means that observers must be able to discriminate situations in which it is possible to stop from situations in which it is no longer possible to stop. To make such discriminations across variations in size and speed, observers must be able to detect and calibrate information about ideal deceleration. Although an optical variable exists that invariantly specifies ideal deceleration (i.e., GOFR $\times \dot{\theta} / \theta$ ), it is not necessary to assume that the invariant is used by all observers at all times (as it is for the tau-dot model). Affordance-based control allows for the possibility that actors could rely on the invariant or on one of the infinite number of noninvariants and that actors might learn to use more reliable optical variables with practice.

The affordance-based model offers several advantages over the tau-dot model. First, it explains the origin of size and speed effects that have been reported in this and other studies on braking (e.g., Andersen et al., 1999; Andersen \& Sauer, 2004), as well as in many other studies on catching, hitting, and collision avoidance. Such findings are inconsistent with the tau-dot model because $\dot{\tau}$ is invariant across changes in size and speed. According to the model proposed here, these effects arise when observers rely on optical variables that are not invariant over changes in size and/or speed, such as those that were used by the subjects (particularly inexperienced ones) in Experiments 1 and 2 .

Second, the affordance-based model allows us to attribute improvement in performance to a process of perceptual attunement to more reliable information. In studies of continuously controlled visually guided actions such as braking, the role of attunement is almost always neglected because the single optical invariant assumption underlies most models of such actions. Thus, rejection of the single optical invariant assumption and acceptance of the affordance-based model opens the door for the study of perceptual learning in visually guided action. Such learning was revealed in the present study as subjects converged from $\dot{\theta} / \theta$ toward GOFR $\times \dot{\theta} / \theta$ with practice.

Lastly, previous studies on braking have revealed that performance is generally good in the absence of a textured ground plane (Yilmaz \& Warren, 1995). Because $\dot{\tau}$ is independent of optic flow from the ground surface, this result has been interpreted as evidence that braking is controlled on the basis of $\dot{\tau}$. However, performance was slightly better when the ground plane was present, especially when initial time-to-contact was short (Fajen, 2005a). This result is difficult to explain if observers rely on $\dot{\tau}$ but is unsurprising if braking is controlled on the basis of information about ideal deceleration. In the absence of the ground plane to provide GOFR, observers are forced to rely on a noninvariant. In the present study, subjects who performed the task in the absence of the ground plane relied on $\dot{\theta} / \theta$. They were still able to perform the task, but not as well as the subjects in the ground condition, in which the invariant was available. Thus, unlike the tau-dot model, the affordance-based model explains why performance is good when the ground plane is absent but better when the ground plane is present.
In summary, the results of the present study suggest that observers (especially novices) rely on noninvariants to guide braking and that they can learn to rely on more effective optical variables with practice. These findings raise questions about the single optical invariant assumption, upon which the tau-dot model of braking is based. Although the present study was focused on the task of braking, models of other continuously controlled, visually guided actions (e.g., steering, fly ball catching, intercepting moving targets) are also built upon the single optical invariant assumption. Perceptual attunement is likely to play an important role in learning to perform these tasks as well.

\section{AUTHOR NOTE}

This research was supported by Grant BCS 0236734 from the National Science Foundation. Correspondence about this article should be addressed to B. R. Fajen, Department of Cognitive Science, Rensselaer Polytechnic Institute, Carnegie Building 308, 110 8th Street, Troy, NY 12180 (e-mail: fajenb@rpi.edu).

\section{REFERENCES}

Andersen, G. J., Cisneros, J., Atchley, P., \& Saidpour, A. (1999). Speed, size, and edge-rate information for the detection of collision events. Journal of Experimental Psychology: Human Perception \& Performance, 25, 256-269.

Andersen, G. J., \& Sauer, C. W. (2004). Optical information for collision detection during deceleration. In H. Hecht \& G. J. P. Savelsbergh (Eds.), Time-to-contact (pp. 93-108). Amsterdam: Elsevier.

BARDY, B. G., \& WARREN, W. H., JR. (1997). Visual control of braking in goal-directed action and sport. Journal of Sports Sciences, 15, 607-620.

Chapman, S. (1968). Catching a baseball. American Journal of Physics, 36, 368-370.

Chardenon, A., Montagne, G., Buekers, M. J., \& Laurent, M. (2002). The visual control of ball interception during human locomotion. Neuroscience Letters, 334, 13-16.

DeLuCia, P. R., \& WARREN, R. (1994). Pictorial and motion-based depth information during active control of self-motion: Size-arrival effects on collision avoidance. Journal of Experimental Psychology: Human Perception \& Performance, 20, 783-798.

FAJEN, B. R. (2005a). Calibration, information, and control strategies for braking to avoid a collision. Journal of Experimental Psychology: Human Perception \& Performance, 31, 480-501.

FAJEN, B. R. (2005b). Perceiving possibilities for action: On the necessity of calibration and perceptual learning for the visual guidance of action. Perception, 34, 717-740.

FAJEN, B. R. (2005c). The scaling of information to action in visually guided braking. Journal of Experimental Psychology: Human Perception \& Performance, 31, 1107-1123.

FAJEN, B. R. (2007). Affordance-based control of visually guided action. Ecological Psychology, 19, 383-410.

FAJEN, B. R., \& DEVANEY, M. C. (2006). Learning to control collisions: The role of perceptual attunement and action boundaries. Journal of Experimental Psychology: Human Perception \& Performance, 32, 300-313.

FAJEN, B. R., \& WARREN, W. H. (2004). Visual guidance of intercepting a moving target on foot. Perception, 33, 689-715.

JACOBS, D. M., \& MichaELS, C. F. (2006). Lateral interception: I. Operative optical variables, attunement, and calibration. Journal of Experimental Psychology: Human Perception \& Performance, 32, 443-458.

Jacobs, D. M., Runeson, S., \& Michaels, C. F. (2001). Learning to visually perceive the relative mass of colliding balls in globally and locally constrained task ecologies. Journal of Experimental Psychology: Human Perception \& Performance, 27, 1019-1038.

LEE, D. N. (1976). A theory of visual control of braking based on information about time-to-collision. Perception, 5, 437-459.

Lenoir, M., Musch, E., Janssens, M., Thiery, E., \& Uyttenhove, J. (1999). Intercepting moving objects during self-motion. Journal of Motor Behavior, 31, 55-67. 
McBeath, M. K., Shaffer, D. M., \& Kaiser, M. K. (1995). How baseball outfielders determine where to run to catch fly balls. Science, 268, 569-573.

Michaels, C. F., Zeinstra, E. B., \& Oudejans, R. R. D. (2001). Information and action in punching a falling ball. Quarterly Journal of Experimental Psychology, 54A, 69-93.

Montagne, G., Laurent, M., Durey, A., \& Bootsma, R. [J.] (1999). Movement reversals in ball catching. Experimental Brain Research, 129, 87-92.

Oudejans, R. R. D., Michaels, C. F., Bakker, F. C., \& Davids, K. (1999). Shedding some light on catching in the dark: Perceptual mechanisms for catching fly balls. Journal of Experimental Psychology: Human Perception \& Performance, 25, 531-542.

Peper, L., Bootsma, R. J., Mestre, D. R., \& Bakker, F. C. (1994) Catching balls: How to get the hand to the right place at the right time. Journal of Experimental Psychology: Human Perception \& Performance, 20, 591-612.

Smith, M. R. H., Flach, J. M., Dittman, S. M., \& Stanard, T. (2001). Monocular optical constraints on collision control. Journal of Experimental Psychology: Human Perception \& Performance, 27, 395-410.

VAn der Kamp, J., Savelsbergh, G. [J. P.], \& Smeets, J. (1997). Multiple information sources in interceptive timing. Human Movement Science, 16, 787-821.

VAn Hof, P., van der Kamp, J., \& Savelsbergh, G. J. P. (2004). The information-based control of interceptive timing: A developmental perspective. In H. Hecht \& G. J. P. Savelsbergh (Eds.), Time-to-contact (pp. 141-171). Amsterdam: Elsevier.

VAN Hof, P., VAN DER KAMP, J., \& SAVElsbergh, G. J. P. (2006). Threeto eight-month-old infants' catching under monocular and binocular vision. Human Movement Science, 25, 18-36.

WANN, J. [P.], \& LAND, M. (2000). Steering with or without the flow: Is the retrieval of heading necessary? Trends in Cognitive Sciences, 4, 319-324
WanN, J. P., \& Swapp, D. K. (2000). Why you should look where you are going. Nature Neuroscience, 3, 647-648.

WARREN, R. (1982). Optical transformations during movement: Review of the optical concomitants of egospeed. Columbus: Ohio State University, Department of Psychology.

Withagen, R., \& Michaels, C. F. (2005). The role of feedback information for calibration and attunement in perceiving length by dynamic touch. Journal of Experimental Psychology: Human Perception \& Performance, 31, 1379-1390.

Yilmaz, E. H., \& WARREN, W. H., JR. (1995). Visual control of braking: A test of the $\dot{\tau}$ hypothesis. Journal of Experimental Psychology: Human Perception \& Performance, 21, 996-1014.

\section{NOTES}

1. Speed is also specified by edge rate (ER), which is the number of texture elements that pass by a fixed reference in the visual field per unit time (Warren, 1982). However, Fajen (2005a) found that GOFR had a stronger influence on braking behavior than did ER. Therefore, I will refer to GOFR, rather than ER, throughout this article.

2. For undershoots, the required change in deceleration was calculated by taking the difference between the actual deceleration at the beginning of the adjustment and the ideal deceleration at the end of the adjustment. For overshoots, ideal deceleration at the overshoot frame, rather than the end of the adjustment, was used, because ideal deceleration reverses direction at the overshoot frame.

3. Data from 1 subject in the air condition were eliminated because this subject did not follow instructions.

4. Data from 1 subject in the ground condition and 1 subject in the air condition were excluded because these subjects pumped the brakes despite instructions to make smooth brake adjustments.

(Manuscript received September 16, 2005; revision accepted for publication March 8, 2008.) 